\title{
Elusive light switch
}

Writing in Cell, Jin and colleagues report that they have identified the long sought-after isomerase enzyme responsible for the regeneration of the visual pigment rhodopsin in the retina after light exposure.

Light perception in vertebrates is mediated by rhodopsin, which is embedded in the disc membrane of rod cells. Rhodopsin is composed of a protein portion, opsin, which is a member of the G-proteincoupled receptor family, and a light-absorbing portion, 11-cisretinaldehyde, which is a derivative of vitamin A.

Activation of rhodopsin starts with the absorption of light by 11cis-retinaldehyde, which is then converted to all-trans-retinaldehyde and subsequently dissociates from opsin through a process known as photobleaching. Opsin itself is insensitive to light, and the regeneration of rhodopsin depends on a cascade of enzymatic reactions that converts all-trans-retinaldehyde back to 11-cis-retinaldehyde, completing the visual cycle. The key step in this cycle is the conversion of all-trans-retinyl palmitate into 11-cis-retinol, which is catalysed by an isomerase that has eluded researchers for more than two decades.

In this study, Jin et al. carried out an unbiased functional screen and discovered that RPE65 (retinal pigment epithelium-specific protein 65), which was thought to present the substrate to the elusive enzyme, was the isomerase itself. To confirm this finding, RPE65 was expressed in mammalian and insect cells alone or in combination with other enzymes of the visual cycle. RPE65 alone was sufficient to catalyse the conversion of all-trans-retinyl palmitate into all-cis-retinol, which indicates that RPE65 has intrinsic isomerase activity. This conclusion is also supported by in vitro isomerase assays using membrane extracts from cells expressing RPE65.

Mutations in the RPE65 gene are associated with Leber congenital amaurosis, an recessively inherited disease that is believed to cause up to $20 \%$ of all cases of childhood blindness. The researchers used site-directed mutagenesis to generate alleles of RPE65 associated with the disease, and found that cells expressing these mutants could not convert 11-trans-retinyl palmitate into 11-cis-retinol. The protein levels of wild-type and mutated RPE65 were similar in these cells, which indicates that lack of 11-cis-retinol production was due to reduced catalytic activity of RPE65 rather than a defect in protein expression or stability.

These findings have solved the long-standing mystery about the identity of the retinoid isomerase, and have significant implications for treating congenital blindness.

Jane Qiu

(2) References and links ORIGINAL ReSEARCh PAPER Jin, M. et al. Rpe65 is the retinoid isomerase in bovine retinal pigment epithelium. Cell 122, 449-459 (2005) FURTHER READING Arshavsky, $V$. Y. et al. G proteins and phototransduction. Annu. Rev. Physiol. 64, 153-178 (2002)

\section{WEB SITE}

Travis' laboratory: http://dgsom.healthsciences. ucla.edu/institution/personnel?personnel $i d=45691$

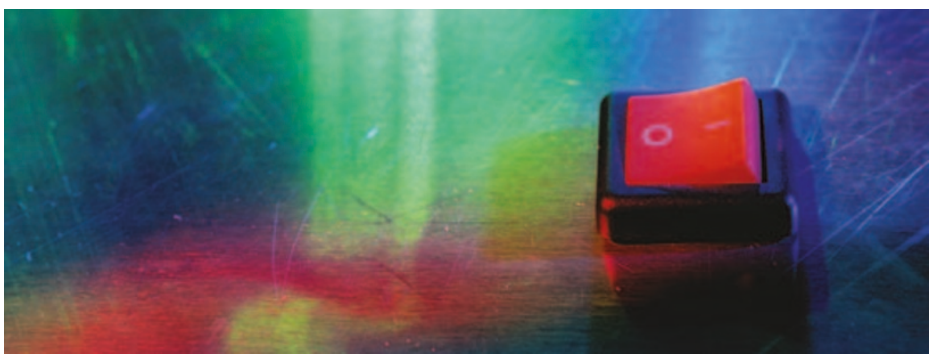

\section{IN BRIEF}

\section{SOCIAL COGNITION}

The V1a vasopressin receptor is necessary and sufficient for normal social recognition: a gene replacement study.

Bielsky, I. F. et al. Neuron 47, 503-513 (2005)

A deficiency in the vasopressin receptor $\mathrm{V} 1 \mathrm{aR}$ in mice has important consequences for social recognition and anxietyrelated behaviour. Both the lateral septum and the medial amygdala have been implicated in these behaviours. In this study, V1aR antagonists in wild-type mice led to social impairments when targeted to the lateral septum but not the medial amygdala. Moreover, re-expressing V1aR in the lateral septum of mice deficient in V1aR led to complete rescue of social recognition behaviours, confirming a crucial role for this region in complex social behaviour.

\section{NEUROLOGICAL DISORDERS}

Amyloid precursor protein promotes post-developmental neurite arborization in the Drosophila brain.

Leyssen, M. et al. EMBO J. 24, 2944-2955 (2005)

Amyloid precursor protein (APP) is well known to be involved in the pathogenesis of Alzheimer's disease. Studies of the expression of human and Drosophila forms of APP in the Drosophila brain now provide clues that APP is also important for axonal outgrowth in the adult but not in the developing brain, and that increased expression of APP is triggered specifically in response to traumatic brain injury.

\section{GLIA}

Synaptic transmission onto hippocampal glial cells with hGFAP promoter activity.

Jabs, R. et al. J. Cell Sci. 118, 3791-3803 (2005)

There is growing evidence that glial cells work as communication elements in the brain. Now, a distinct type of glial cell in the hippocampus - dubbed the GluR cell - has been shown in mice to have synapse-like structures. The researchers present the first evidence that these cells receive both stimulus-correlated and spontaneous GABA ( $\gamma$-aminobutyric acid)- and glutamatemediated input, thereby revealing novel signalling mechanisms between neurons and glia.

\section{DEVELOPMENT}

The Notch ligands DLL1 and JAG2 act synergistically to regulate hair cell development in the mammalian inner ear.

Kiernan, A. E. et al. Development 132, 4353-4362 (2005)

The number of sensory hair cells that form in the organ of Corti in the mammalian inner ear is thought to be regulated by Notch signalling. In mouse embryos, two Notch ligands - Dll1 and Jag2 - seemed to interact synergistically to regulate hair cell differentiation in the cochlea. Results indicated that Notch pathways acted through both lateral inhibition - when a cell differentiating as one type prevents neighbouring cells from differentiating to the same type - and the control of cell proliferation levels. 